\title{
Potential Threat of Tracheal Diverticulum to Thermal Ablation Treatment of Thyroid Nodule
}

\author{
Jianquan Zhang, $M D^{a, c, *}$, Lei Yan, $M D^{b}$, Zongping Diao, $\mathrm{MD}^{c}$, Hongqiong Chen, $\mathrm{MD}^{a}$, Jie Cheng, MD ${ }^{a}$ \\ ${ }^{a}$ Department of Ultrasound, Super-minimally Invasive Surgery, Shanghai International Medical Center, Shanghai, China; ${ }^{b}$ Department \\ of Medical Ultrasound, The 100th Hospital of PLA, Suzhou City, China; ${ }^{c}$ Department of Medical Ultrasound, Shanghai Changzheng \\ Hospital, Shanghai, China
}

Received October 8, 2018; revision requested February 8, 2019; revision received November 18, 2018; accepted November 20.

\begin{abstract}
Application of thermal ablation therapy by using microwave or radiofrequency energy for benign thyroid nodules or/ and thyroid malignancies gets increased world-wide. Potential risks involved in the ablation procedure are not uncommon and could be catastrophic when out of control. Ablation for pharyngeal esophageal diverticulum's (PED) being misdiagnosed as thyroid nodules constitutes the risks. In this case report, the authors presented another potential threat to such ablation treatment, which derived from a trachea diverticulum complicated with inflammatory hyperplastic tissue by misdiagnosis as thyroid nodules. The tracheal diverticulum (TD) reported in this study was interpreted as a calcified thyroid nodule on ultrasonography at first, then microwave ablation (MWA) was accordingly recommended as one of the therapeutic options. However, the first author, experienced with the ultrasonic features of PED, insisted the differential diagnosis should be made between nodule and PED. Subsequently, swallow contrast-enhanced ultrasonography (CEUS) and barium meal test were carried out successively, but neither of them reported the presence of PED. Percutaneous fine-needle aspiration (FNA) was hence operated to identify pathological characteristics of the "calcified thyroid nodule", and cytological tests under microscopy indicated the nodule originated from trachea due to the presence of ciliated columnar epithelial cells with inflammatory exudates within the specimen. Cervical and thoracic X-ray CT examination was further conducted, and a TD was discovered. The case was finally concluded as a TD with inflammatory hyperplastic tissue. The value of this case lies in that once a TD was misdiagnosed as ablation-candidate thyroid nodule in conventional ultrasonography could cause the latent danger to thyroid thermal therapy. Rigorous and effective differential diagnosis prior to thermal ablation procedure could prevent misdiagnosis and mistreatment.
\end{abstract}

Key words: Tracheal diverticulum; Pharyngeal esophageal diverticulum; Thyroid nodule; Thermal therapy; Microwave ablation

Advanced Ultrasound in Diagnosis and Therapy 2019;01:006-011

DOI: 10.37015/AUDT.2019.190802

$\mathrm{T}$ hermal ablation therapy under real-time ultrasound guidance has been used for minimally invasive management of thyroid nodules increasingly. As the anatomical complexities of the neck compartments, it is critical to determine the origination of the lesions prior to the ablation to improve operational safety. High frequency ultrasonography is considered as front-line screening imaging modality for abnormalities derived from thyroid glands, parathyroid glands, upper segment of esophagus, and regional lymphatic nodes in the neck as well. Tracheal diverticulum (TD) is a small cystic chamber formed by local expansion of the tracheal wall, which contains gas and communicates with the tracheal cavity [1]. Theoretically, a TD has distinct- different ultrasonic features from a thyroid nodule due to the essence of containing abundant gas, and is a condition rarely come across in clinical ultrasound practice, making it unfamiliar to the majority of ultrasonographers. To make matter worse, a pure TD complicated with solid tissue gets 'impure' in ultrasonography and could be similar to a thyroid nodule. Serious iatrogenic injury is inevitable once the TD is misdiagnosed with thyroid nodule and then treated by using microwave ablation (MWA) or radiofrequency ablation (RFA). In present

* Corresponding Author: Department of Ultrasound, Super-minimally Invasive Surgery, Shanghai International Medical Center, 4358 Kangxin Road, Shanghai 201318, China.

e-mail:wintersnow9090@sina.com 
case report, an impure TD simulated a thyroid nodule was recommended for MWA initially, then a series of valid differential diagnosis was carried out to avoid iatrogenic events occur.

\section{Case Report}

\section{Clinical Features}

A 61-year-old woman was transferred to our hospital for frequent nocturnal chest distress and even dyspnea lasting about a year, accompanied with irregular and intermittent attack of stabbing pain in the right supraclavicular fossa. She denied the complaint of cough, sputum, hemoptysis, choking swallowing, and food reflux. She lost 2-Kg weight since the onset symptoms developed.

\section{Neck Ultrasonographic Findings}

Conventional Ultrasonography Prior to admition, she had been informed of a suspicious thyroid nodule in her right neck compartment leading to her clinical symptoms, and was recommended to receive thermal ablation therapy for the target 'thyroid nodule'. On July 18, 2018, a comprehensive ultrasound scanning was performed (11 MHz of probe frequency, Preirus, Hitachi, Japan) on her entire neck areas. The nodule was well-defined in roughly circular shape with a smooth, continuous and echogenic capsule in relatively consistent thickness. The upper margin of the nodule was closely adjacent to the lower pole of right thyroid gland, the anterior border was adjacent to the ostium of the right common carotid artery (CCA), and the medial border was adjacent to the wall of the trachea (Fig. 1).

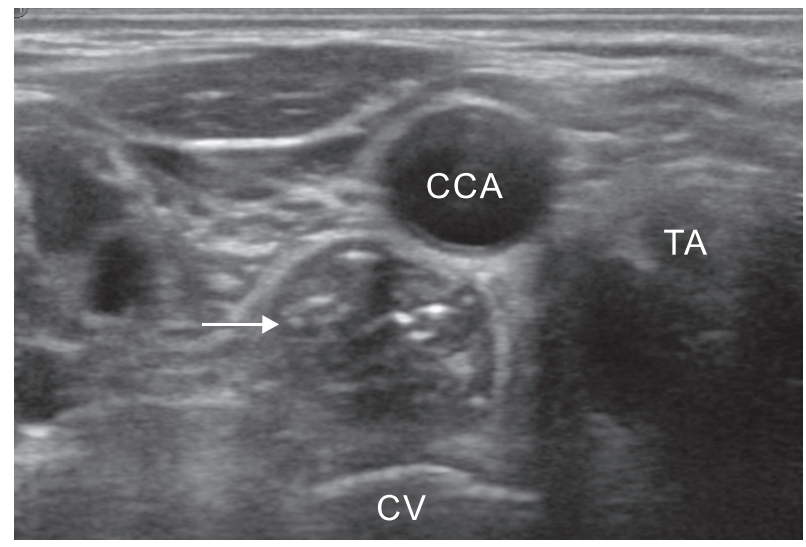

Figure 1 The nodule (arrow indicating) is closely behind the right common carotid artery (CCA), lateral to the trachea (TR), and anterior to the cervical vertebra $(\mathrm{CV})$ on a transverse section.

On the longitudinal section, the nodule manifested in drumstick mushroom-like shape, extending to the mediastinum with getting narrowed down slightly (Fig. 2 ). The nodule was primarily hypoechoic, but with numerous and radially arranged punctate hyperechoic (Fig. 3). Real-time elastic ultrasound showed that the nodule was mainly in dark blue display, indicating a hard texture (Fig. 4). Color Doppler flow imaging (CDFI) demonstrated that flow were partially visible at the capsule and internal hypoechoic areas (Fig. 5). Realtime scanning showed the nodule moved simultaneously with thyroid gland during swallowing. Signs of free air migration were not presented inside the nodule. The conventional ultrasonography strongly indicated that the nodule was a pharyngeal esophageal diverticulum (PED).

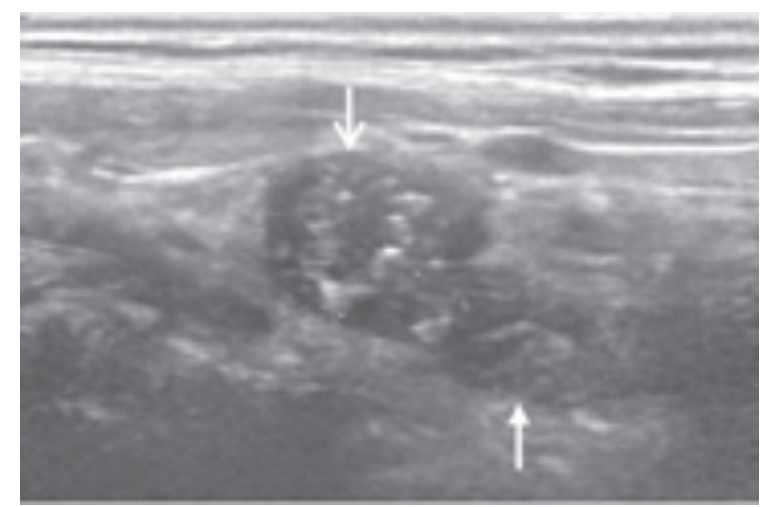

Figure 2 The nodule is larger and roundish in the head section (open arrow indicating) and narrows down (closed arrow indicating) into the mediastinum.

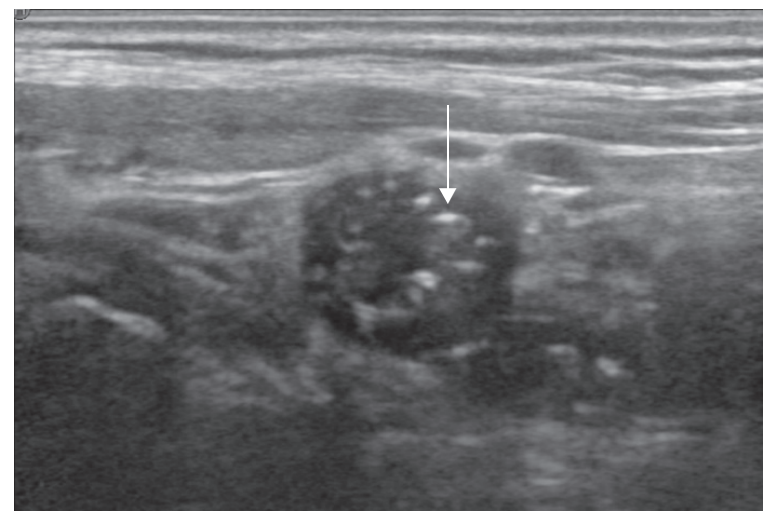

Figure 3 Punctiform strong echoes being caused by air microbubbles is presented in the nodule.

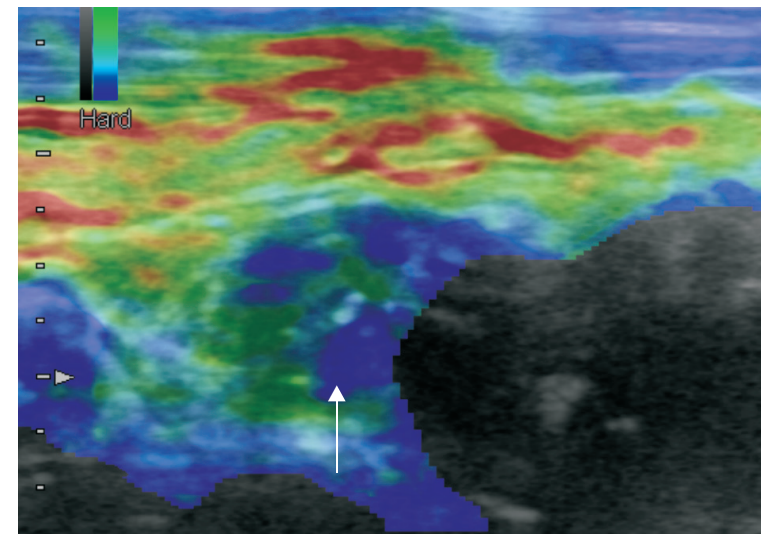

Figure 4 Most area of the nodule appears in blue on real-time elastography. 


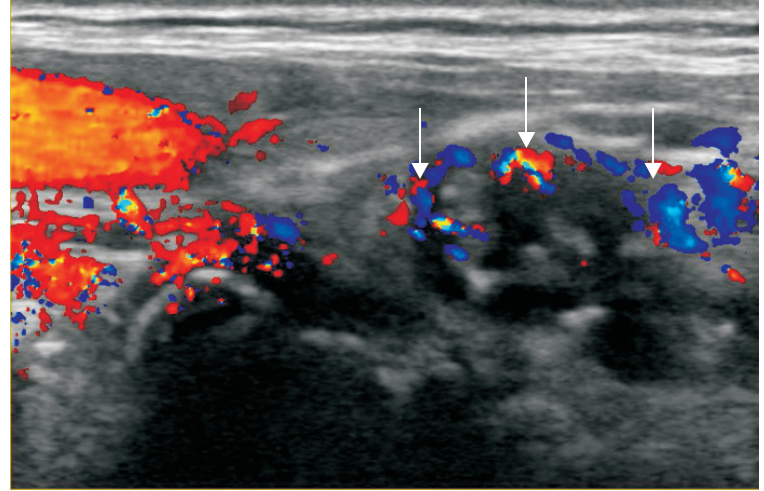

Figure 5 There are fruitful color signals of blood flow at the capsular area and the marginal area of the nodule.

Swallow oral Contrast-enhanced Ultrasonography For further differential diagnosis, swallow contrast-enhanced ultrasonography (CEUS) was performed by using Logic E8 apparatus (GE Company, America). The low-frequency probe conventionally used for abdominal scanning was adopted to improve the visibility of the contrast agents instead of the high-frequency probe. Hyper-echo from the contrast agents passing through the esophageal cavity were clearly detected, however, enhancement in the area corresponding to the nodule was absent and therefore PED as diagnosis for the nodule was denied (Fig. 6). Due to PED is a contraindication for thermal ablation, the patient was transferred to receive an oral barium meal X-ray examination for further validation. The result of barium meal examination excluded PED, which is consistent with the swallow CEUS (Fig. 7).

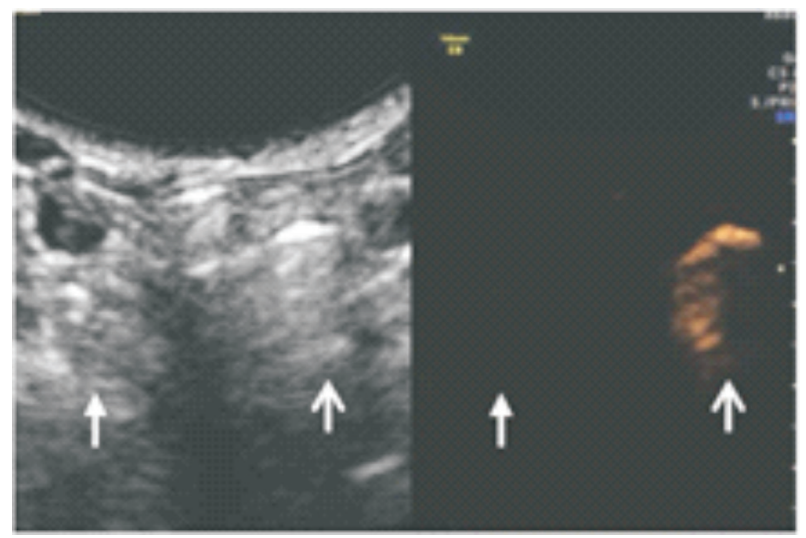

Figure 6 On CEUS-mode image (right side), there are strong echoes from the contrast agents in the area of esophagus cavity (open arrow indicating), while there are no echoes in the area corresponding to the nodule (closed arrow indicating).

\section{Ultrasound-guided Percutaneous Biopsy}

Core-needle Biopsy (CNB) The patient urged to take microwave ablation treatment to relieve symptoms, on

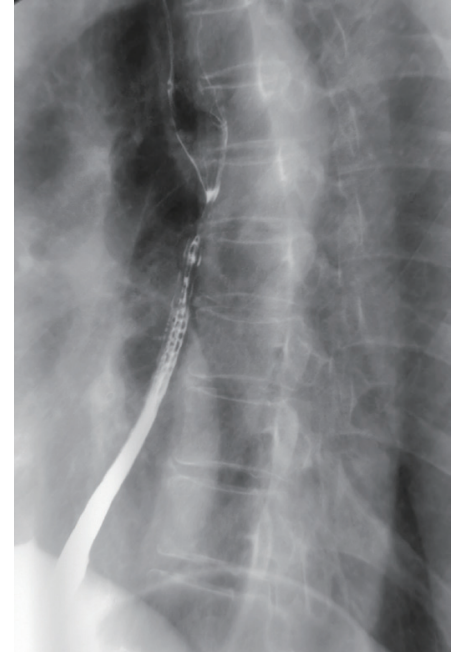

Figure 7 There is no manifestation of esophageal diverticulum on barium meal X-ray examination

another hand, ultrasound-guided biopsy for preoperative pathological diagnosis was accordingly proposed was implemented on July 20, 2018 since the nodule was excluded to originate from PED. Due to the complex anatomical position of the nodule, hydro-dissection was conducted for building a safe needling approach to protect right common carotid artery, trachea, external jugular vein and internal jugular vein in case of accidental injury. During this procedure, the nodule was found to be tightly adhesive to the right lateral wall of trachea and separation was failed. However, the nodule was readily separated from the other adjacent tissues. Moreover, the capsule of the nodule was so tough that 18-gauge core needle tip failed to puncture, and CNB was unimplemented as a result.

Fine-needle Aspiration (FNA) A 23-guage FNA was administrated instead of CNB and amount of cytological specimen was obtained successfully. Rapid cytological examination reported a small amount of ciliated columnar epithelial cells originating from tracheal mucosa, besides numerous inflammatory cells with exudates in the aspirates (Fig. 8), which suggested that the nodule was derived from the trachea.
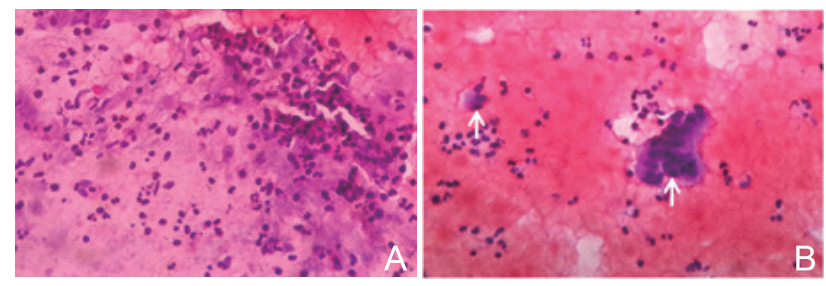

Figure 8 Cytological findings from the FNA specimen $(\times 40)$ (A) there is plenty of inflammatory cells with exudates; (B) Ciliated columnar epithelial cells (arrows) appear in the specimen 


\section{Neck and Chest CT Examination}

Contrast-enhanced CT (CECT) evaluation was applied subsequently for detailing the anatomic relationships between the nodule and trachea. CECT demonstrated a circular soft nodular lesion measured approximately $11 \mathrm{~mm} \times 13 \mathrm{~mm}$ in size and located right lateral-posterior to the trachea at the entrance of the thoracic cavity. The lesion contained solid tissue in slight enhancement as well as microbubbles of air density. A small cystic lesion was seen at the posterior wall of the trachea and communicated with the tracheal cavity (Fig. 9), indicated the nodule was a TD with inflammatory soft tissue.

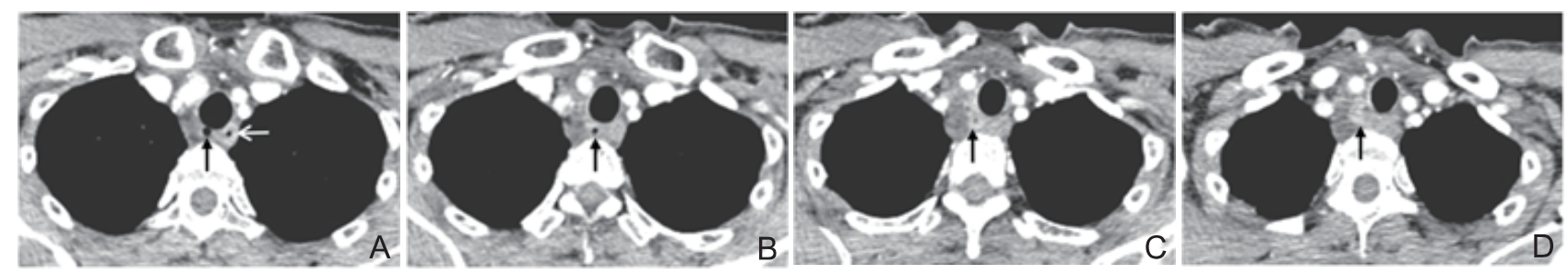

Figure 9 CT images of the nodulea. (A) Tiny diverticulum (closed arrow indicating) communicating with the trachea passage at the posterior wall. The open arrow indicates the esophagus passage. (B) Tiny gas-filling structure (arrow indicates) seen posterior to the trachea at an upper level is postulated to be the neck of the diverticulum. (C) The slice of the lower edge of the nodule (arrow indicates) shows slight enhancement and contains air microbubbles density. (D) The slice of the mid-portion of the nodule shows enhancement and air microbubbles.

\section{Results}

\section{Suspicion and exclusion of PED by ultrasound examination}

On conventional gray-scale ultrasonograms, the suspicious thyroid nodule had similar ultrasonic features to PED, representing oval or round shape, well-defined margin, heterogeneous echoic internal textures with radially arranged hyperechoic punctuates, and the specific location close to trachea. A definite confirmation should be made whether the nodule was a PED or not. Swallow oral CEUS excluded PED then further confirmed by a following barium meal X-ray test.

\section{FNA cytological evidence for suspicion of TD}

Upon the exclusion of PED, the nodule was supposed to be eligible for microwave ablation, and the preablative percutaneous biopsy was necessary to conduct.

Generally, it was difficult for core biopsy needle to puncture a thyroid nodule with thick and well-calcified capsule or densely calcified tissue. Even ultrasonogram showed this nodule was not severely calcified, the core needle failed to break through the capsule, indicating the unusual toughness of the nodule capsule. Although fine needle puncture was difficult as well, the aspiration was finally accessible. Cytological study disclosed the presence of cellular components of trachea suggesting the nodule originated from trachea. It also reminded us the so-called nodule capsule was virtually the tracheal cartilage.

\section{CT evidence for imaging diagnosis of tracheal diverticulum}

A neck and chest CT scanning was appropriate for examine the overview and details of the nodule. On successive CT slices, a distinct TD was detected closely below the nodule, and a tiny air-filling passage was present between them. The nodule appeared in solid tissue value compound with small areas of air.

After comprehensive differential diagnosis, the nodule initially considered as thyroid lesion was finally diagnosed with TD complicated with inflammatory tissue hyperplasia. Since thermal ablation therapy is contraindicated for the condition, the patient was advised to consult surgeons for surgical operation afterwards.

\section{Discussion}

Ultrasound scanning is normally inadequate for detecting TD, however, features of a solid nodule with or without air manifestation could help when the diverticulum is superficially located and complicated with hyperplastic and inflammatory soft tissue [2]. This situation would probably lead to misdiagnosis as thyroid nodule and even mistreatment by thermal ablation therapy, which has been progressively operated in clinical practice for treating thyroid nodules or parathyroid nodules [3]. In recent years, thermal ablation therapy by using microwave or/and radiofrequency energy has emerged an increasing application for treatment of benign thyroid nodules and papillary thyroid microcarcinoma due to eutherapeutic effect with less invasiveness [4-7]. However, various lesions in the neck area are similar to thyroid nodules on ultrasonography, thus undervaluation of these lesions would increase the risk of thermal ablation therapies, such as PED [810]. TD involved in thyroid ablation treatment is lack of evidence to date in the previous publications. 
Normally, containing air makes TD could be differentiated with thyroid nodules in the acoustic manifestations, however when the mucosa layer of TD emerges inflammatory hyperplasia, the diverticulum turns to be a partially or completely solid nodule while gas component decreases, and. In the present study, her diverticulum in consolidation was adjacent to the thyroid that it was misunderstood as a thyroid nodule. More seriously, her first attending physician made an incorrect treatment recommendation to use microwave ablation.

In this patient, inflammatory hyperplasia tissue and exudates filled the majority of the TD, while gas within it was only a few scattered small bubbles. Accordingly, the ultrasonic features of the nodule were massively hypoechoic accompanied by dot-like hyper-echoes.

Such echo manifestation is different from simple TD but is similar to PED, due to the latter contains gas and inflammatory hyperplasia tissue as well. Thus, we were led to the first consideration of the nodule as a potential PED, and corresponding differential diagnostic evaluations were taken.

Barium meal X-ray is a conventional method for the diagnosis of PED [11]. Although the contrast agents and imaging modalities are different, swallow CEUS and barium meal have similar diagnostic principles for PED. As a new mode of ultrasonography, swallow CEUS helps to achieve a quick and definite differential diagnosis of PED and has played an important role in preventing malpractice as thyroid nodule for receiving microwave ablation therapy [12-13].

High-frequency probes in most of the diagnostic ultrasound equipment made before the early 2010's is inefficient in displaying contrast agents passing through the superficial organs. Therefore, we succeeded in raising the display power of contrast agents passing through the superficial structures by using abdominalscanning purposed low-frequency probe [14]. But in this case, swallow CEUS with low-frequency probe failed to indicate any of characteristic CEUS manifestations of PED. Nevertheless, we applied for a barium meal examination for further validation the presence of PED, since the possibility of PED must be ruled out to avoid misdiagnosis and treatment due to the potential defects of a single inspection method.

Thymic tumors are not uncommon [15], there is a possibility of mistaking thymic tumor as thyroid nodule due to the location of the thymus is high or even close to the thyroid gland in some cases. To date, we still lack experience on whether thymic tumor is suitable for ablative treatment, so it is necessary to obtain a definite histopathologic diagnosis before taking any therapy. Percutaneous core needle biopsy is essential and benefit to achieve the goal. Once the neck nodule is identified as a retrosternal nodular goiter through biopsy, MWA is feasible and valuable in treatment.

During the biopsy, we obtained two unusual experiences which were different from the most cervical nodules. First, the nodule was closely attached to the trachea wall, and the hydro-dissection maneuver was unable to them. Second, the capsule of the nodules was too tough to break through with a 18 -gauge Tru-cut biopsy needle. Then a 23 -gauge fine-needle biopsy was conducted instead. The rapid cytological examination revealed ciliated columnar epithelium of tracheal mucosa as well as numerous inflammatory cells and exudates, suggesting the possibility that the target nodule was a TD combined with inflammatory tissue. Immediate cervical and chest X-ray contrast-enhanced CT examination indicated a slight enhancement in the peripheral of the nodule consistent with CDFI. In addition, a small TD was detected at the mid-posterior wall and an anatomical association between the nodule and the small diverticulum could be observed by successive slice. The most likely diagnosis with the nodule was a TD combined with inflammatory tissue hyperplasia on CT.

The clinical symptoms of this patient may be related to inflammatory hyperplasia of TD mucosa. It is reasonable to anticipate that $t$ both severity of inflammatory response in the diverticulum and accumulation of inflammatory exudates may directly lead to the patient's chest tile-like pain.

Thermal ablation therapy could be beneficial to thyroid-nodule patients with certain therapeutic effect, minimal tissue damage and good repeatability. However, this therapy applicated in clinical practice just recently and most physicians in the field have not been familiar with it. Thus, it is important to follow the indications and implement the standard procedure strictly, particularly an accurate ultrasound diagnosis of a certain neck nodule is critical and should be treated scrupulously.

\section{Conclusion}

TD complicated with inflammatory hyperplastic tissue could be similar to a calcified thyroid nodule on ultrasonographic images. Thermal therapy using MWA or RFA would be incorrectly recommended as treatment protocol when lack of evaluation. Being highly cautious with this rare condition and making adequate differential diagnosis are essential to assure a safe procedure of thyroid thermal ablation.

\section{Conflicts of Interest}

The authors declare that there is no conflict of interest. 


\section{References}

[1] Tanrivermis Sayit A, Elmali M, Saglam D, Celenk C. The diseases of airway-tracheal diverticulum: a review of the literature. J Thorac Dis 2016;8:E1163-E1167.

[2] Kurt A, Sayit AT, Ipek A, Tatar IG. A multi detector computed tomography survey of tracheal diverticulum. Eurasian J Med 2013;45:145-148.

[3] Teng D, Sui G, Liu C, Wang Y, Xia Y, Wang H. Long-term efficacy of ultrasound-guided low power microwave ablation for the treatment of primary papillary thyroid microcarcinoma: a 3-year follow-up study. J Cancer Res Clin Oncol 2018;144:771-779.

[4] Che Y, Jin S, Shi C, Wang L, Zhang X, Li Y, et al. Treatment of Benign Thyroid Nodules: Comparison of Surgery with Radiofrequency Ablation. AJNR Am J Neuroradiol 2015;36:13211325.

[5] Hamidi O, Callstrom MR, Lee RA, Dean D, Castro MR, Morris JC, et al. Outcomes of Radiofrequency Ablation Therapy for Large Benign Thyroid Nodules: A Mayo Clinic Case Series. Mayo Clin Proc 2018;93:1018-1025.

[6] Li J, Liu Y, Liu J, Qian L. Ultrasound-guided percutaneous microwave ablation versus surgery for papillary thyroid microcarcinoma. Int J Hyperthermia 2018;34:653-659.

[7] Kim JH, Baek JH, Sung JY, Min HS, Kim KW, Hah JH, et al. Radiofrequency ablation of low-risk small papillary thyroidcarcinoma: preliminary results for patients ineligible for surgery. Int J Hyperthermia 2017;22;33:212-219.
[8] Achille G, Castellana M, Russo S, Montepara M, Giagulli VA, Triggiani V. Zenker Diverticulum: A Potential Pitfall in Thyroid Ultrasound Evaluation: A Case Report and Systematic Review of Literature. Endocr Metab Immune Disord Drug Targets 2019;19:9599.

[9] Lixin J, Bing H, Zhigang W, Binghui Z. Sonographic diagnosis features of Zenker diverticulum. Eur J Radiol 2011;80:e13-e19.

[10] Zhang JQ, Yan L, Chen HQ. Microwave ablation of thyroid nodules complicated with Zenker's diverticulum: Case report. Chin J Interv Imaging Ther 2018;15:48.

[11] Bagheri R, Maddah G, Mashhadi MR, Haghi SZ, Tavassoli A, Ghamari MJ, et al. Esophageal diverticula: Analysis of 25 cases. Asian Cardiovasc Thorac Ann 2014;22:583-587.

[12] Cui XW, Ignee A, Baum U, Dietrich CF. Feasibility and usefulness of using swallow contrast-enhanced ultrasound to diagnose Zenker's diverticulum: preliminary results. Ultrasound Med Biol 2015;41:975981.

[13] Zhang JQ, Chen HQ, Yan L. Potential threat of pharyngo-esophageal diverticulum to thermal ablation treatment of thyroid nodule and rapid differential diagnosis through swallow contrast-enhanced ultrasonography. Chin J Ultrasonogr 2019;28:35-39.

[14] Zhang JQ. High-frequency contrast-enhanced ultrasound in evaluation of perfusion of superficial lesions: limitations and countermeasures. Acad J Sec Mil Med Univ 2007;28:1193-1196.

[15] Bushan K, Sharma S, Verma H. A review of thymic tumors. Indian J Surg Oncol 2013;4:112-116. 